OPEN ACCESS

Edited by:

Andrea Ladányi,

National Institute of Oncology (NIO),

Hungary

*Correspondence:

Young Kyung Bae

ykbae@ynu.ac.kr

https://orcid.org/0000-0002-6689-

9413

Received: 04 March 2021 Accepted: 16 June 2021

Published: 29 June 2021

Citation:

Park MH, Choi JE, Kim J-R and Bae YK (2021) Immunohistochemical Expressions of SenescenceAssociated Secretory Phenotype and

Its Association With Immune

Microenvironments and Clinicopathological Factors in Invasive Breast Cancer.

Pathol. Oncol. Res. 27:1609795.

doi: 10.3389/pore.2021.1609795

\section{Immunohistochemical Expressions of Senescence-Associated Secretory Phenotype and Its Association With Immune Microenvironments and Clinicopathological Factors in Invasive Breast Cancer}

\author{
Min Hui Park ${ }^{1}$, Jung Eun Choi ${ }^{2}$, Jae-Ryong Kim ${ }^{3}$ and Young Kyung Bae ${ }^{1 *}$ \\ ${ }^{1}$ Department of Pathology, Yeungnam University College of Medicine, Daegu, South Korea, ${ }^{2}$ Department of Surgery, Division of \\ Breast Surgery, Yeungnam University College of Medicine, Daegu, South Korea, ${ }^{3}$ Department of Biochemistry and Molecular \\ Biology, Yeungnam University College of Medicine, Daegu, South Korea
}

This study was undertaken to investigate immunohistochemical expression of the senescence-associated secretory phenotype (SASP) in invasive breast cancer (IBC) tissues and to determine relationships between SASP positivity and tumor microenvironments and the clinicopathological characteristics of IBC. Immunohistochemistry for senescence markers, that is, high mobility group box-1 (HMGB1), p16, p15, and decoy receptor 2 (DCR2), was performed in tissue microarrays of 1140 IBC samples. Cases positive for at least one of these four markers were considered SASP-positive. Relations between SASP and tumor characteristics, including immune microenvironments (stromal tumor-infiltrating lymphocytes [sTILs] density and numbers of intraepithelial CD103-positive [iCD103 + ] lymphocytes) and clinical outcomes were retrospectively evaluated. HMGB1, p16, p15, or DCR2 was positive in $6.7 \%, 26.6 \%, 21.1 \%$, and $26.5 \%$, respectively, of the 1,140 cases. Six hundred and five $(53.1 \%)$ cases were SASP positive, and SASP positivity was significantly associated with histologic grade 3, high-sTIL and iCD103 + lymphocyte counts, absence of ER or PR, and a high Ki-67 index. Although SASP did not predict breast cancer-specific survival (BCSS) or disease-free survival (DFS) in the entire cohort, SASP positivity in luminal A IBC was associated with poor BCSS and DFS. However, patients with SASP-positive TNBC showed better survival than those with SASP-negative TNBC. In multivariate analysis, SASP positivity was an independent prognostic factor in both luminal A IBC and TNBC, although the effect on prognosis was the opposite. In conclusion, SASP would be involved in the modulation of immune microenvironments and tumor progression in IBC, and its prognostic significance depends on molecular subtype.

Keywords: immunohistochemistry, breast cancer, senescence, prognosis, markers 


\section{INTRODUCTION}

Internal and external stimuli can damage DNA, and when this damage cannot be repaired, cells undergo aging (senescence) or apoptosis. Senescent cells secrete inflammatory cytokines, chemokines, growth factors and matrix metalloproteinases (MMPs) and modulate their microenvironments, which is called senescence-associated secretory phenotype (SASP) and can take various cell-dependent forms [1-3].

Tumor cell senescence can be induced by oncogenes, radiotherapy, or chemotherapy, and senescent tumor cells that acquire SASP change tumor microenvironments. Furthermore, SASP factors are known to display anti-tumor effects by inducing the senescence of surrounding tumor cells through paracrine or autocrine mechanisms or by activating the host immune system to remove tumor cells [4-6]. However, SASP can also promote angiogenesis, proliferation, and epithelial-mesenchymal transition (EMT), which result in tumor progression and invasion [7]. Furthermore, cancer cells exhibiting SASP cause chronic inflammation, tissue fibrosis, and increase tumor cell drug resistance [4].

The senescence-associated markers that are usually used to identify SASP in tumor tissue samples include p16, p15, p53, ADP-ribosylation factor (ARF), p21, differentially expressed in chondrocytes protein 1 (DEC1), decoy receptor 2 (DCR2), and high mobility group box 1 (HMGB1) [8-12]. p16 inhibits cell division by regulating $\mathrm{G} 1$ to $\mathrm{S}$ transition, and loss of $\mathrm{p} 16$ function promotes cell cycle progression and is involved in the pathogenesis of several cancers [13,14]. In addition, p16 is upregulated during tissue aging [15] and has been considered a prognostic marker in cancer patients [16-18]. p15 regulates G1 progression and is upregulated by senescence-associated $\beta$-galactosidase (SA- $\beta$-Gal, a classic SASP marker) activation [9]. p15 also participates in growth arrest in Ras-transformed NIH 3T3 fibroblasts [19].

DCR2 is a TRAIL (tumor necrosis factor-related apoptosisinducing ligand) receptor and inhibits TRAIL-induced apoptosis in response to chemotherapy [20]. HMGB1 is a member of the alarmin family and an important component of tissue damage signals and also inhibits apoptosis by reducing the functions of caspase-3 and -9 [21-23]. HMGB1 is translocated from nuclei to cytoplasm and extracellular space in senescent cells, and this promotes the release of SASP factors, such as interleukin- $1 \beta$ (IL$1 \beta)$, IL-6, and MMP3 [8,10,11]. Therefore, loss of nuclear expression of HMGB1 has been used for identifying senescent cells $[10,24,25]$.

Several studies have addressed SASP marker expression in Korea and overseas. p15, p16, and DCR2 were reported to be upregulated during prostate cancer progression [12], and DCR2 expression was increased in lung cancer cell lines treated with anticancer drugs [20]. In addition, HMGB1 upregulation has been observed in breast cancer [26], colon cancer [22] and gastrointestinal stromal tumor [27] tissues. However, few studies have shown combined expression of senescence markers and its association with clinicopathologic features in breast cancer [28].

In the present study, we used immunohistochemistry (IHC) to determine the expressions of senescence-associated markers in invasive breast cancer (IBC) tissues and then investigated the effects of SASP on tumor microenvironments, clinicopathological characteristics, and patient prognosis.

\section{MATERIALS AND METHODS}

\section{Collection of Breast Cancer Tissues and Clinicopathological Data}

IBC tissue samples surgically resected at Yeungnam University Medical Center from 1995 to 2007 were included in this study. Patients that received neoadjuvant chemotherapy or with a microinvasive carcinoma were excluded. Clinicopathological information and follow-up data were collected from medical records including pathology reports. Breast cancer-specific survival (BCSS) was defined as time interval between surgical resection and death from -related cause or last follow-up. Disease-free survival (DFS) was defined as time interval between surgical resection and tumor relapse (locoregional recurrence or distant metastasis), death or last follow-up.

This study was approved by the Institutional Review Board (IRB) of Yeungnam University Medical Center (YUMC2019-10002), which waived the requirement for informed consent.

\section{Tissue Microarrays and Immunohistochemical Staining for Senescence-Associated Markers}

For this study, we used tissue microarray (TMA) blocks that have been used in our previous studies [29,30]. To briefly explain TMA construction, a pair of $1.5 \mathrm{~mm}$-diameter tissue cores was retrieved from a representative tumor block using a manual tissue microarrayer (Quick-Ray ${ }^{\circledR}$, Unitma, Seoul, Korea), and consecutively transferred to the recipient blocks (Unitma). A total of 38 TMA blocks representing 1518 IBC cases were created.

IHC staining for HMGB1, p16, p15, and DCR2 was performed on TMA sections using a Benchmark XT immunostainer (Ventana Medical Systems) as described in Table 1. All IHC slides were interpreted by two observers (YKB and MHP) under a multi-headed microscope. HMGB1 was ubiquitously expressed in nuclei of most cells including non-neoplastic and cancer cells. Therefore, loss of nuclear staining or faint nuclear staining compared with internal control (non-neoplastic epithelial cells, fibroblasts, endothelial cells, or inflammatory cells) in $>50 \%$ of tumor cells was considered positive for HMGB1. Immunoreactivity for p16, p15, or DCR2 was not observed in both nuclei and cytoplasm of normal breast epithelial cells. For these markers, intensities of cytoplasmic staining and proportions of immunoreactive tumor cells were evaluated. Staining intensities were scored as follows: 0 (no staining), 1 (weak), 2 (moderate), and 3 (strong). Proportions of immunoreactive tumor cells were expressed as percentages. IHC scores were generated by multiplying intensity scores by percentages of immunoreactive tumor cells, which resulted in a range of $0-300$. We used 75 th percentile IHC scores as cut-off values to define positivity for $\mathrm{p} 16, \mathrm{p} 15$, and DCR2. Cases positive for at 
TABLE 1 | Antibodies and staining conditions used in the study.

\begin{tabular}{|c|c|c|c|c|c|}
\hline Antibody & Source & Clone & Dilution & Antigen retrieval & Incubation time \\
\hline HMGB1 & Abcam & EPR3507 & $1: 400$ & Mild $^{\mathrm{a}}, \mathrm{CC} 1$ & 40 min, RT \\
\hline p15 & Abcam & Polyclonal & $1: 200$ & Autoclave, 10min & $10 \mathrm{~h}, 4^{\circ} \mathrm{C}$ \\
\hline p16 & Ventana & $\mathrm{E} 6 \mathrm{H} 4$ & Prediluted & Standard $^{\mathrm{b}}, \mathrm{CC} 1$ & $16 \mathrm{~min}, 37^{\circ} \mathrm{C}$ \\
\hline DCR2 & Abcam & EPR3588(2) & $1: 250$ & Standard $^{\mathrm{b}}, \mathrm{CC} 1$ & $40 \mathrm{~min}, \mathrm{RT}$ \\
\hline CD103 & Abcam & EPR4166(2) & $1: 500$ & Mild $^{\mathrm{a}}, \mathrm{CC1}$ & $40 \mathrm{~min}, \mathrm{RT}$ \\
\hline
\end{tabular}

CC1, cell conditioning 1 solution; DCR2, decoy receptor 2; HMGB1, high mobility group box-1; RT, room temperature.

${ }^{a}$ Mild antigen retrieval condition was performed for $30 \mathrm{~min}$ at $100^{\circ} \mathrm{C}$.

${ }^{b}$ The standard condition was $60 \mathrm{~min}$ at $100^{\circ} \mathrm{C}$.

These procedures were performed using a Benchmark ${ }^{\circledR} X T$ autoimmunostainer.

least one of the four markers were considered SASP-positive tumors.

\section{Molecular Classification of Invasive Breast Cancer}

In order to apply consistent criteria on determining estrogen receptor (ER), progesterone receptor (PR) and human epidermal growth factor receptor 2 (HER2) statuses, we repeated ER, PR, and HER2 studies on TMA sections, as described in our previous study [31] and their results were interpreted according to the latest guidelines [32,33].

$\mathrm{Ki}-67$ indices were reported at diagnosis and expressed as percentages of positive cells per 500-1,000 tumor cells.

Definition used for surrogate molecular subtypes of IBC was as follows [34]; luminal A (ER-positive/PR-positive/HER2negative/Ki-67 $\leq$ 20\%), HER2-negative luminal B1 (ERpositive/HER2-negative/Ki-67 > 20\% or ER-positive/HER2negative/PR-negative or low), HER2-positive luminal B2 (ERpositive/HER2-positive/any Ki-67/any PR), HER2-positive (ERnegative/PR-negative/HER2-positive), or triple-negative (TNBC) (ER-negative/PR-negative/HER2-negative). A low PR status was defined as an Allred score of $<5$.

\section{Tumor Microenvironment: Stromal Tumor-Infiltrating Lymphocyte and Intraepithelial CD103-Positive (iCD103+) Lymphocyte Measurements}

The stromal tumor-infiltrating lymphocyte (sTIL) density and intraepithelial CD103 - positive (iCD103 + ) lymphocyte count for each case were obtained from our previous studies conducted in the same IBC cohort [35,30]. Brief descriptions of the research methods are as follows. sTIL densities were defined as percentages of total intratumoral stromal areas infiltrated with lymphocytes and plasma cells and measured on a whole section HandE slide for each case [36]. Under low magnification observations (X100), average sTIL densities were presented as; $0-1 \%, 2-5 \%, 6-10 \%$, $11 \%-20 \%, 21-30 \%$, or further $10 \%$ increments [35]. iCD 103 + lymphocytes were defined as CD103 + lymphocytes in tumor cell nests or CD103 + lymphocytes adhering to tumor cells when tumors exhibited highly infiltrative growth $[37,38]$. IHC for CD103 [EPR4166(2), 1:500, Cambridge, United Kingdom] was performed using TMA sections [30], and stained slides were scanned using an Aperio CS2 slide scanner (Leica Biosystems, Nussloch, Germany). In an area captured at $\times 200$ magnification $\left(0.45 \mathrm{~mm}^{2}\right)$ with the highest CD103 + lymphocyte density, total numbers of iCD103 + lymphocytes were manually counted and converted into numbers per $1 \mathrm{~mm}^{2}$.

\section{Statistical Analysis}

Statistical analysis was conducted using SPSS (Version 23.0 for Windows, IBM, Armonk, NY, United States). The significance of correlations between SASP and patient characteristics was determined using chi-square test. Survival curves were plotted using the Kaplan-Meier method and the significance of survival differences between groups was determined using the log-rank test. Variables found to be significant by univariate analyses were subjected to Cox regression proportional hazard analysis. Adjusted hazard ratios and associated 95\% confidence intervals were calculated for each variable. A $p$ value of $<0.05$ was considered statistically significant.

\section{RESULTS}

\section{Clinicopathological Characteristics of Cases}

Of the 1518 IBC samples included in the TMAs, only 1,140 yielded informative IHC results for the four SASP markers (HMGB1, p16, p15, and DCR2). The other 378 samples were exhausted by prior use, lost while performing immunosistochemical staining, or included ductal carcinoma in situ, extensive necrosis, or non-neoplastic tissue rather than viable invasive tumor.

Patients ranged in age from 20 to 86 years (mean, 48 years), and tumor sizes were $0.5-11.0 \mathrm{~cm}$ (mean, $2.4 \mathrm{~cm}$ ). Axillary lymph node metastasis was present in $544(47.8 \%)$, and lymphovascular invasion (LVI) in 600 (52.6\%). Histological grades were 1 in 178 (15.6\%), 2 in 326 (28.6\%), and 3 in 636 (55.8\%) patients (Table 2).

sTIL density could be obtained in 1,135 of 1,140 cases and $\leq 1 \%$ in $554(48.8 \%)$ cases, $2-5 \%$ in $244(21.5 \%), 6-10 \%$ in $125(11 \%)$, $11-20 \%$ in $76(6.7 \%), 21-30 \%$ in $40(3.5 \%), 31-40 \%$ in $26(2.3 \%)$, 
TABLE 2 | Relationships between the senescence-associated secretory phenotype (SASP) and clinicopathological characteristics in patients with invasive breast carcinoma.

\begin{tabular}{|c|c|c|c|c|c|c|c|c|c|c|c|c|}
\hline \multirow[t]{2}{*}{ Characteristics } & \multicolumn{3}{|c|}{ All patients, $\mathbf{N}(\%)$} & \multirow[t]{2}{*}{$p$-value } & \multicolumn{3}{|c|}{ Luminal A, N (\%) } & \multirow[t]{2}{*}{$p$-value } & \multicolumn{3}{|c|}{ Triple-negative, N (\%) } & \multirow[t]{2}{*}{$p$-value } \\
\hline & No & SASP (-) & SASP (+) & & No & SASP (-) & SASP (+) & & No & SASP (-) & SASP (+) & \\
\hline Age & & & & 0.239 & & & & 0.245 & & & & $0.01^{\star}$ \\
\hline$<50$ & 713 & $325(45.6)$ & $388(54.4)$ & & 207 & $133(64.3)$ & $74(35.7)$ & & 153 & $22(14.4)$ & $131(85.6)$ & \\
\hline$\geq 50$ & 427 & 210 (49.2) & 217 (50.8) & & 96 & $55(57.3)$ & $41(42.7)$ & & 94 & $26(27.7)$ & 68 (72.3) & \\
\hline Tumor size & & & & 0.1 & & & & 0.951 & & & & 0.03 \\
\hline$\leq 2 \mathrm{~cm}$ & 565 & 279 (49.4) & $286(50.6)$ & & 193 & $120(62.2)$ & 73 (37.8) & & 101 & 13 (12.9) & $88(87.1)$ & \\
\hline$>2 \mathrm{~cm}$ & 575 & $256(44.5)$ & $319(55.5)$ & & 110 & $68(61.8)$ & 42 (38.2) & & 146 & $35(24)$ & $111(76)$ & \\
\hline LN metastasis $^{a}$ & & & & 0.163 & & & & 0.31 & & & & 0.088 \\
\hline Absent & 594 & 267 (44.9) & 327 (55.1) & & 167 & $108(64.7)$ & 59 (35.3) & & 155 & $25(16.1)$ & $130(83.9)$ & \\
\hline Present & 544 & 267 (49.1) & 277 (50.9) & & 134 & 79 (59) & $55(41)$ & & 92 & $23(25)$ & $69(75)$ & \\
\hline LVI & & & & 0.445 & & & & 0.008 & & & & 0.019 \\
\hline Absent & 540 & $247(45.7)$ & 293 (54.3) & & 148 & $103(69.6)$ & $45(30.4)$ & & 150 & $22(14.7)$ & $128(85.3)$ & \\
\hline Present & 600 & $288(48)$ & $312(52)$ & & 155 & $85(54.8)$ & 70 (45.2) & & 97 & $26(26.8)$ & $71(73.2)$ & \\
\hline Histologic grade & & & & $<0.001$ & & & & 0.113 & & & & 0.001 \\
\hline 1 and 2 & 504 & 301 (59.7) & 203 (40.3) & & 267 & $170(63.7)$ & 97 (36.3) & & 22 & $10(45.5)$ & $12(54.5)$ & \\
\hline 3 & 636 & $234(36.8)$ & 402 (63.2) & & 36 & $18(50)$ & $18(50)$ & & 225 & 38 (16.9) & $187(83.1)$ & \\
\hline Stromal TILs ${ }^{b}$ & & & & $<0.001$ & & & & 0.117 & & & & 0.981 \\
\hline Low $(\leq 5 \%)$ & 798 & $423(53)$ & $375(47)$ & & 274 & $174(63.5)$ & $100(36.5)$ & & 108 & $21(19.4)$ & 87 (80.6) & \\
\hline High (> 5\%) & 337 & $110(32.6)$ & $227(67.4)$ & & 27 & $13(48.1)$ & $14(51.9)$ & & 138 & 27 (19.6) & $111(80.4)$ & \\
\hline iCD103 + lymphocyte & & & & $<0.001$ & & & & 0.869 & & & & 0.042 \\
\hline Low $\left(<38 / \mathrm{mm}^{2}\right)$ & 820 & 417 (50.9) & $403(49.1)$ & & 252 & $153(60.7)$ & 99 (39.3) & & 122 & $29(23.8)$ & 93 (76.2) & \\
\hline $\operatorname{High}\left(\geq 38 / \mathrm{mm}^{2}\right)$ & 212 & $58(27.4)$ & $154(72.6)$ & & 12 & 7 (58.3) & $5(41.7)$ & & 106 & $14(13.2)$ & $92(86.8)$ & \\
\hline ER & & & & $<0.001$ & & & & & & & & \\
\hline Positive & 764 & $434(56.8)$ & $330(43.2)$ & & & & & & & & & \\
\hline Negative & 376 & 101 (26.9) & $275(73.1)$ & & & & & & & & & \\
\hline PR & & & & $<0.001$ & & & & & & & & \\
\hline Positive & 645 & $368(57.1)$ & 277 (42.9) & & & & & & & & & \\
\hline Negative & 495 & $167(33.7)$ & 328 (66.3) & & & & & & & & & \\
\hline HER2 & & & & 0.882 & & & & & & & & \\
\hline Negative & 912 & 427 (46.8) & 485 (53.2) & & & & & & & & & \\
\hline Positive & 228 & $108(47.4)$ & 120 (52.6) & & & & & & & & & \\
\hline Ki-67 index ${ }^{d}$ & & & & $<0.001$ & & & & & & & & \\
\hline$\leq 20 \%$ & 444 & $258(58.1)$ & 186 (41.9) & & & & & & & & & \\
\hline$>20 \%$ & 687 & $275(40)$ & $412(60)$ & & & & & & & & & \\
\hline Molecular subtype & & & & $<0.001$ & & & & & & & & \\
\hline Luminal A & 303 & $188(62)$ & $115(38)$ & & & & & & & & & \\
\hline Luminal B1 & 362 & $191(52.8)$ & 171 (47.2) & & & & & & & & & \\
\hline Luminal B2 & 100 & $55(55)$ & $45(45)$ & & & & & & & & & \\
\hline HER2-positive & 128 & $53(41.4)$ & 75 (58.6) & & & & & & & & & \\
\hline TNBC & 247 & 48 (19.4) & 199 (80.6) & & & & & & & & & \\
\hline
\end{tabular}

ER, estrogen receptor; HER2, human epidermal growth factor receptor 2; iCD103 + lymphocyte, intraepithelial CD103-positive lymphocyte; LN, lymph node; LVI, lymphovascular invasion; TILs, tumor-infiltrating lymphocytes; TNBC, triple-negative breast cancer; PR, progesterone receptor.

${ }^{a}$ Two patients did not undergo sentinel lymph node biopsy or axillary lymph node dissection.

${ }^{b}$ Stromal TILs densities were not available in five patients.

${ }^{c}$ iCD103 + lymphocyte counts were missing in 108 patients.

${ }^{d}$ Nine patients did not have a Ki-67 labeling index in their pathology reports.

${ }^{*} p$-values in bold, statistically significant.

$41-50 \%$ in $27(2.4 \%), 51-60 \%$ in $20(1.8 \%), 61-70 \%$ in $13(1.1 \%)$, $71-80 \%$ in $8(0.7 \%)$, and $81-90 \%$ in $2(0.2 \%)$ (Figure 1). For statistical analysis, cases were divided into low- and high-sTIL groups, based on our previous study [35]. As a result, 798 (70.3\%) cases were allocated to the low-sTIL $(\leq 5 \%)$ group and 337 $(29.7 \%)$ to the high-sTIL (>5\%) group.

iCD103 + lymphocyte counts were available in 1,032 of 1,140 cases and ranged from 0 to 1,111 per $\mathrm{mm}^{2}$ of tumor area (Figure 2). Cases were dichotomized into high $\left(\geq 38 / \mathrm{mm}^{2}\right)$ and low $(<38 /$ $\mathrm{mm}^{2}$ ) iCD103 + groups, as we previously described [30]. As a result, $820(79.5 \%)$ belonged to the low-iCD103 + group and 212 $(20.5 \%)$ to the high-iCD103 + group.

Regarding biologic markers of IBC, ER was positive in 764 (67\%), PR was positive in $645(56.6 \%)$ and HER2 positivity (protein overexpression or gene amplification) was observed in 228 (20\%). Ki-67 index was available in 1,131 of 1,140 cases and $>20 \%$ in 687 (60.7\%). As regards molecular subtypes, 303 (26.6\%) cases were classified as luminal A, $362(31.8 \%)$ as luminal B1, 100 $(8.8 \%)$ as luminal B2, $128(11.2 \%)$ as HER2-positive, and 247 $(21.7 \%)$ as triple-negative (TNBC) (Table 2$)$. 

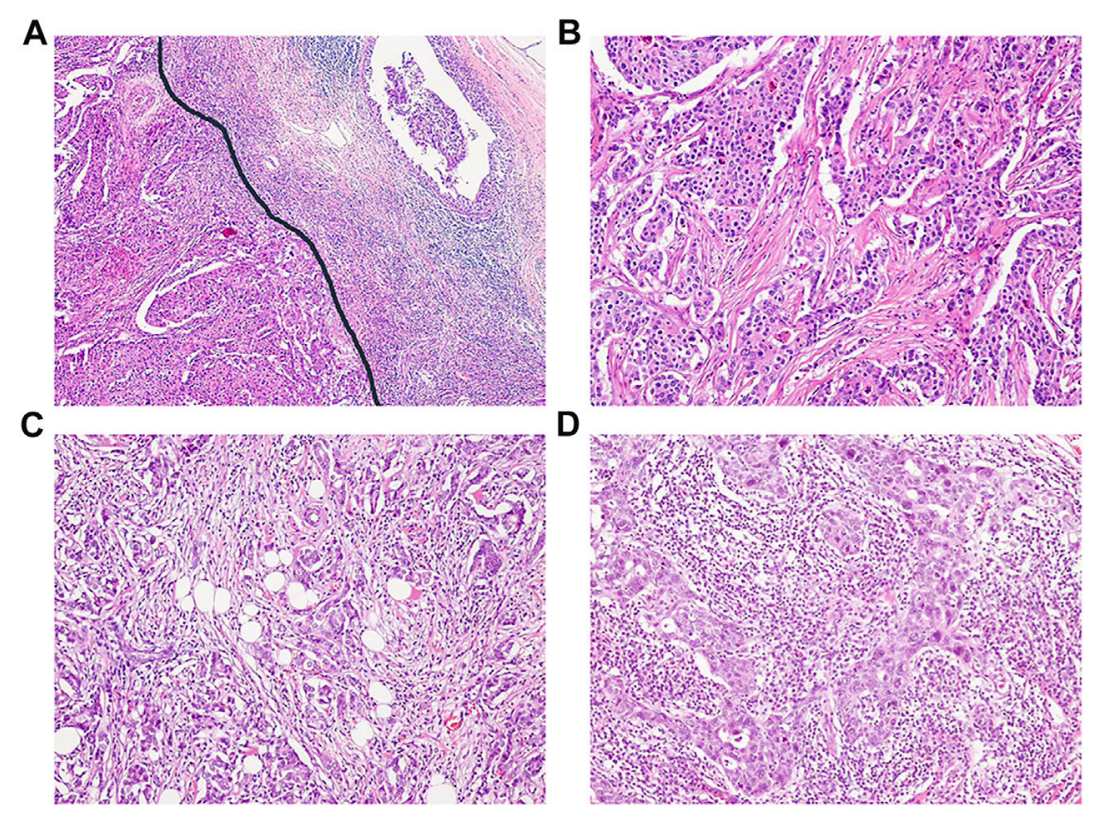

FIGURE 1 | Representative cases with different stromal tumor-infiltrating lymphocyte (sTIL) densities. (A) sTIL densities were measured inside invasive tumor borders (left of the black line) $(\times 40)$. Microscopic findings showing sTIL densities of (B) $1 \%$, (C) $30 \%$, and (D) $80 \%$ (b-d, $\times 100)$.

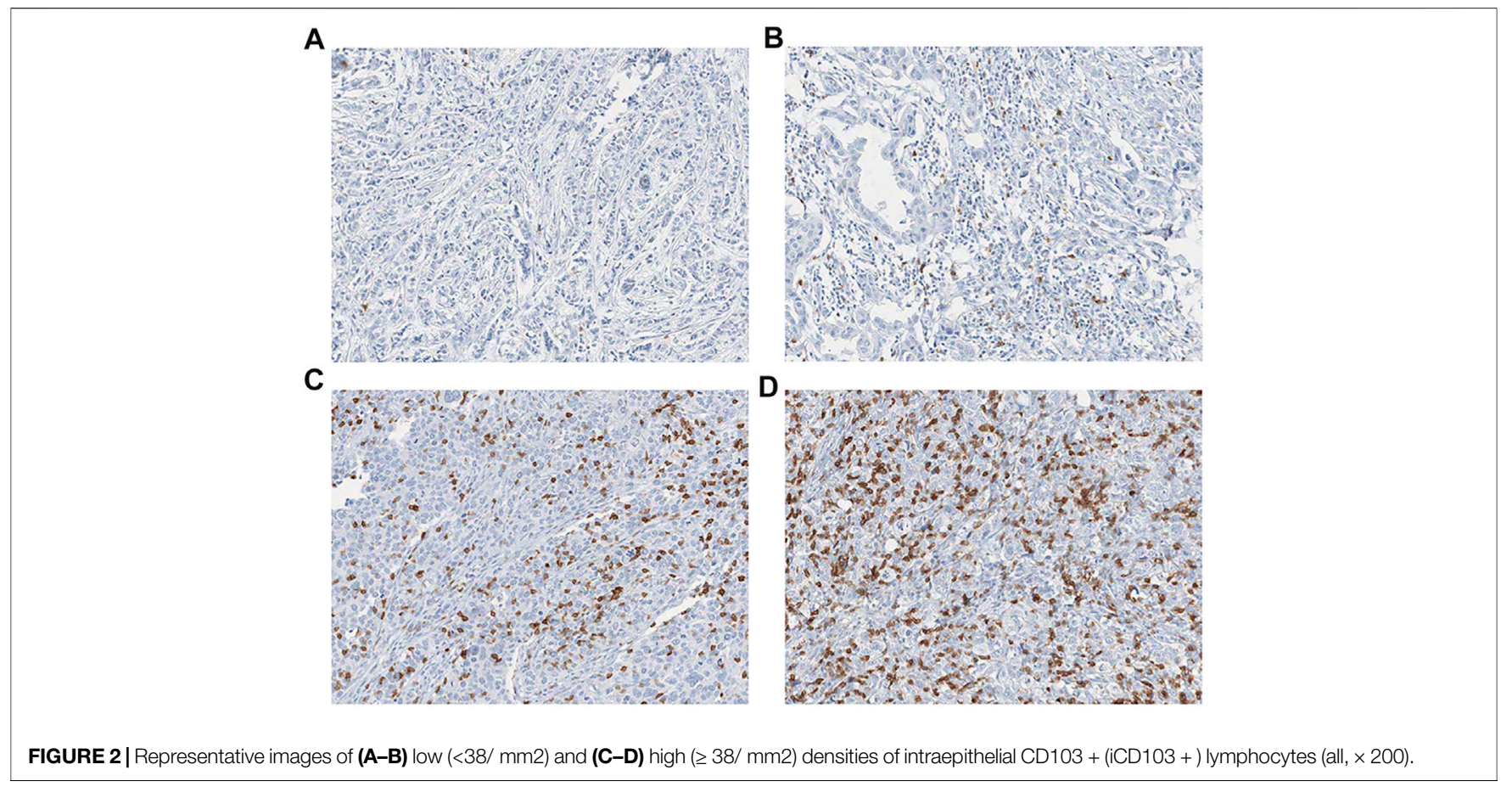

Of the 1,140 patients, $444(38.9 \%)$ received breast-conserving surgery and 696 (61.1\%) mastectomy. For adjuvant therapy, 1,003 $(88 \%)$ received chemotherapy, $545(47.8 \%)$ received radiation, and 772 (67.7\%) received hormone therapy. None of the HER2positive IBC patients received trastuzumab because trastuzumab as an adjuvant regimen was approved in Korea in 2010. The median follow-up period was 133 months (range, 1-277 months), and $212(18.6 \%)$ recurrences and $223(19.6 \%)$ deaths had occurred including 144 breast cancer-related deaths and 79 deaths from other causes. 


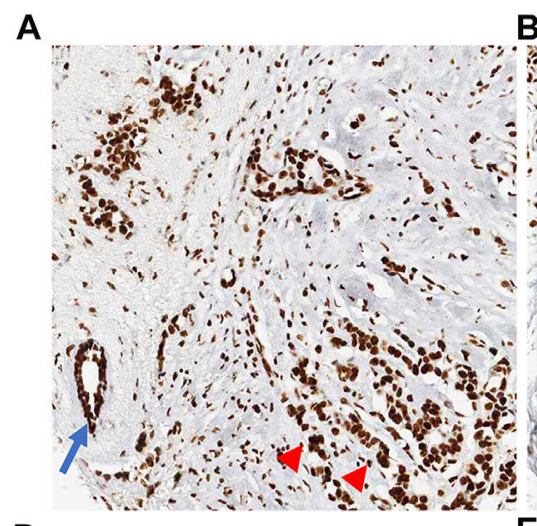

$B$

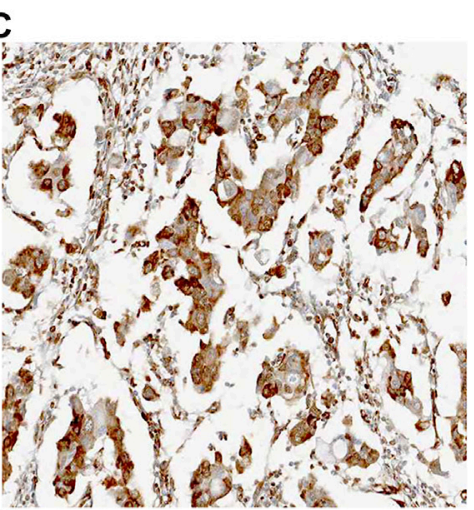

D

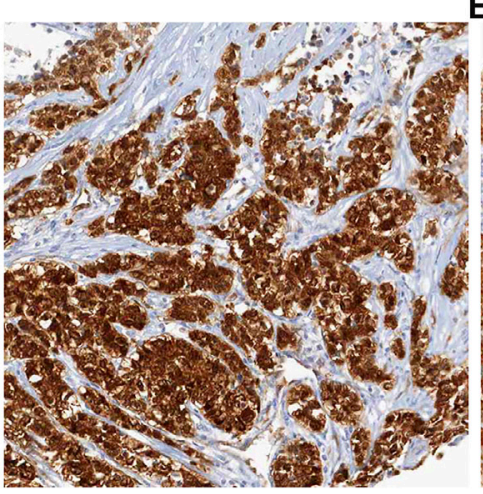

E

$\mathbf{F}$

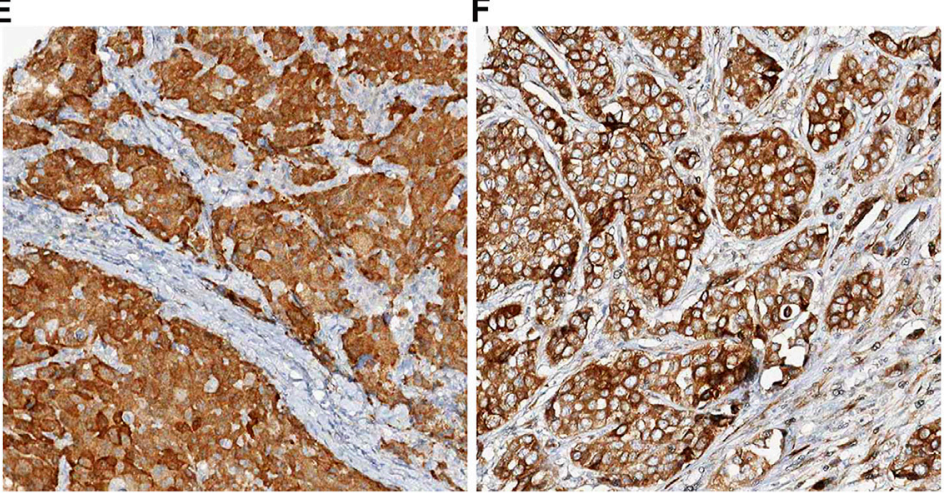

FIGURE 3|Representative images of positive cases for senescence-associated markers. (A) HMGB1 is ubiquitously expressed in all nuclei of non-neoplastic cells (arrow, normal duct) and cancer cells (arrow heads). There is absence of cytoplasmic staining. (B) Positive case for HMGB1. Tumor cells show faint nuclear staining or loss of nuclear staining without cytoplasmic staining (arrow heads). In left upper corner of the image, normal mammary glandular cells show strong nuclear staining for HMGB1 (arrows, internal control). (C) Positive case for HMGB1. This case shows faint nuclear staining with perinuclear cytoplasmic staining for HMGB1. (D) Positive case for $\mathrm{p}$ 16. Tumor cells show diffuse immunoreactivity for $\mathrm{p} 16$ in both cytoplasm and nuclei. (E) Positive case for p15. Most of the nuclei of tumor cells was not stained, only cytoplasm was stained. (F) Positive case for DCR2. Tumor cells show diffuse cytoplasmic staining for DCR2.

\section{Expressions of Senescence-Associated Markers and Relationships Between SASP and Clinicopathological Characteristics}

Seventy-six (6.7\%) cases showed loss of nuclear staining or faint nuclear staining for HMGB1 with or without cytoplasmic staining, therefore they were considered positive for HMGB1. IHC scores corresponding to 75 th percentiles were 30,10 , and 10 for p16, p15, and DCR2, respectively. Of the 1,140 cases, 303 (26.6\%), $240(21.1 \%)$, and 302 (26.5\%) were positive for $\mathrm{p} 16, \mathrm{p} 15$, and DCR2, respectively (Figure 3). As a total, 605 (53.1\%) were positive for at least one senescence-associated marker (362 for one marker, 176 for two markers, 64 for three markers, and three for all four markers) and were regarded as SASP-positive cases. Among the five molecular subtypes, SASP positivity was found in $38 \%$ of luminal A cases, $47.2 \%$ of luminal B1 cases, $45 \%$ of luminal B2 cases, $58.6 \%$ of HER2-positive cases, and $80.6 \%$ of TNBC cases.

Among the 1,140 cases, SASP positivity was significantly associated with histologic grade $(p<0.001)$, sTIL density $(p<$ $0.001)$, iCD103 + lymphocyte count $(p<0.001)$, ER negativity $(p<0.001)$, PR negativity $(p<0.001)$, and a high Ki-67 index $(p<$ 0.001 ) but not with clinicopathological variables, such as age, tumor size, lymph node metastasis, LVI, or HER2 status (Table 2). Furthermore, SASP positivity was significantly greater in HER2-positive IBC and TNBC (Table 2).

When correlations between SASP and clinicopathological features were analyzed for the surrogate molecular subtypes: in the luminal A subtype, SASP was found to be significantly associated with LVI $(p=0.008)$; in the luminal B1 subtype, SASP was significantly associated with an age of $<50(p=0.023)$, a tumor size of $>2 \mathrm{~cm}(p=0.05)$, and high sTILs density $(p=0.024)$; in the luminal B2 subtype, SASP was not associated with any clinicopathological variable; in the HER2-positive subtype, SASP was significantly associated with high iCD103 + lymphocyte count ( $p=0.045)$; and in the TNBC subtype, SASP was significantly associated with an age of $<50(p=0.01)$, a tumor size of $\leq 2 \mathrm{~cm}(p=0.03)$, absence of LVI $(p=0.019)$, high histologic grade $(p=0.001)$, and a high-iCD103+ lymphocyte count ( $p=0.042$ ) (Table 2$)$.

\section{Prognostic Significance of SASP}

Among the 1,140 cases, BCSS and DFS according to presence or absence of SASP were similar $(p=0.851$ and $p=0.341$, respectively) (Figures 4A,B). We performed subgroup analyses 

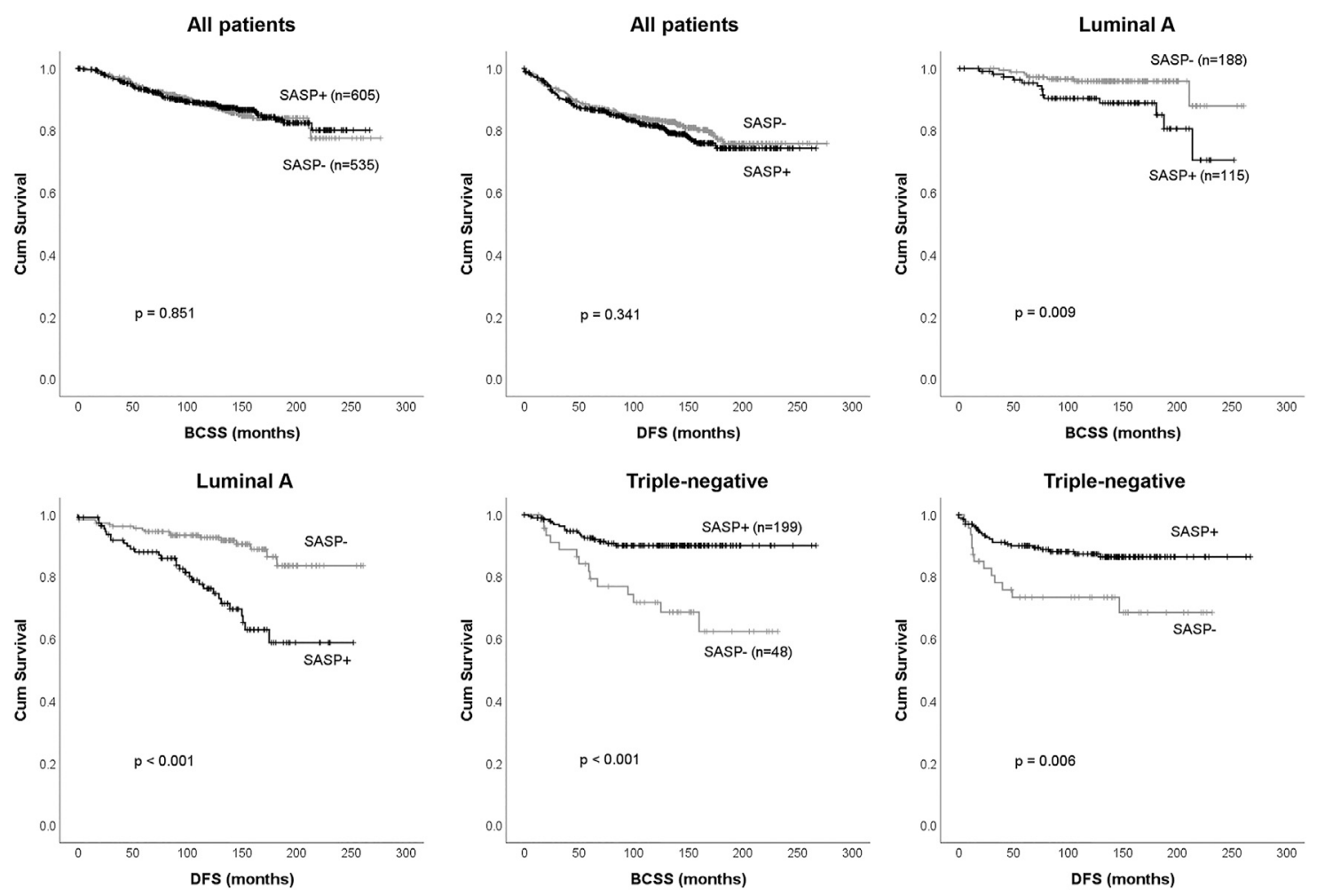

FIGURE 4 | Survival analysis according to presence or absence of SASP. Breast cancer-specific survival (BCSS), and disease-free survival (DFS) for all 1,140 breast cancer patients (A-B) and patients with the luminal A subtype (C-D) or triple-negative breast cancer (E-F).

TABLE 3 | Multivariate analysis of clinicopathological characteristics affecting breast cancer-specific survival and disease-free survival in luminal A and triple-negative breast cancers.

Parameter

\begin{tabular}{cc} 
Breast cancer-specific survival \\
\hline $\begin{array}{c}\text { Hazard } \\
\text { ratio }(95 \% \mathrm{Cl})\end{array}$
\end{tabular}

$2.884(1.204-6.909)$

SASP, present $v s$ absent

LVI, present vs absent

Lymph node metastasis, present vs absent

Histologic grade, 3 vs 1 and 2

Tumor size, $>2 \mathrm{~cm}$ vs $\leq 2 \mathrm{~cm}$

Triple-negative subtype $(n=247)$

SASP, present $v$ s absent

LVI, present vs absent

Lymph node metastasis, present $v s$ absent

iCD103 + lymphocyte, high $\left(\geq 38 / \mathrm{mm}^{2}\right)$ vs low $\left(<38 / \mathrm{mm}^{2}\right)$

Stromal TILs,> 5\% vs $\leq 5 \%$

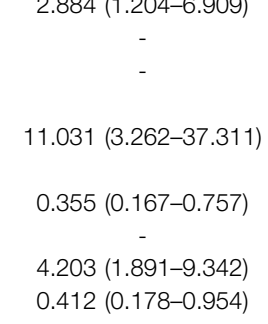

0.017
-
-
$<0.001$
0.007
-
$<0.001$
0.038

$\frac{\text { Disease-free survival }}{\text { Hazard }}$ p-value

$\begin{array}{cc}3.119(1.731-5.618) & <0.001 \\ 1.982(1.072-5.618) & - \\ - & 0.029 \\ & -\end{array}$

0.022 $4.66(2.164-10.035) \quad<0.001$

$0.444(0.222-0.888)$

0.022

Cl, confidence interval; iCD103 + lymphocyte, intraepithelial CD103-positivelymphocyte; LVI, Lymphovascular invasion; SASP, senescence-associated secretory phenotype; TILs, tumorinfiltrating lymphocytes.

to explore the association between SASP and prognosis in different surrogate molecular subtypes. Of the 303 patients with luminal A subtype, those with a SASP-positive tumor had significantly shorter BCSS $(p=0.009)$ and DFS $(p<$ 0.001) (Figures 4 C,D), whereas in TNBC patients, SASP was significantly associated with better BCSS $(p<0.001)$ and DFS $(p=0.006)$ (Figures $\mathbf{4 E}, \mathbf{F})$. However, no such relations were observed for luminal B1, B2 or HER2-positive subtypes.

Multivariate analyses showed SASP independently predicted poor BCSS (HR, 2.884; CI, 1.204-6.909; $p=0.017$ ) and DFS (HR, 
3.119; CI, 1.731-5.618; $p<0.001$ ) for the luminal A subtype, but on the contrary it was an independent predictor of better BCSS (HR, 0.355; CI, 0.167-0.757; $p=0.007$ ) and DFS (HR, 0.437; CI, $0.215-0.889 ; p=0.022$ ) in TNBC (Table 3).

\section{DISCUSSION}

In this study, SASP was found to be significantly associated with surrogate molecular subtype in IBC. SASP positivity was lowest in the luminal A subtype and highest in TNBC. This association concurs with a previous study, which reported that inhibition of ER in ER-expressing cells induced a senescence-like phenotype by increasing SA- $\beta$-Gal activity and decreasing retinoblastoma (RB) protein phosphorylation [39].

Regarding previous studies that investigated the expressions of senescence-associated markers in tumor tissues, Brezniceanu et al. [26] reported that HMGB1 is involved in the regulation of apoptosis and that its overexpression inhibits apoptosis and promotes tumor growth. These researchers also reported that apoptosis was induced by transfection of BAK into the RKO colon cancer cell line and the human and mouse kidney cell lines (203T and NRK1), but that cotransfection of BAK with HMGB1 significantly inhibited apoptosis. In addition, they investigated HMGB1 expression in normal and cancer tissues of the breast by Western blot and IHC and observed that HMGB1 expression was significantly higher in cancer tissues than in normal tissues. In a study on cotransfections of BAK and HMGB1 genes into NRK1 cells, Völp et al. [22] revealed HMGB1 expression inhibited BAK-induced apoptosis by blocking the activities of caspase- 9 and -3 and found that this anti-apoptotic effect was due to inhibitors of apoptosis proteins (IAPs) that bind to and inhibit caspases. HMGB1 overexpression in the RKO cells increased nuclear factor kappa $\mathrm{B}$ (NFkB) activity and resulted in the overexpression of c-IAP2 (a target gene product of $\mathrm{NFkB}$ ). In order to confirm these relationships in tissues, Volp et al. [22] examined the expressions of c-IAP2 and HMGB1 by IHC staining on TMAs of colon cancer and normal colon tissues from 29 patients. They found the expressions of c-IAP2 and HMGB1 were upregulated in $72.4 \%$ of colon cancer tissues, and that the expression of HMGB1 in tumor cell cytoplasm and nuclei was pronounced at tumor borders. Kim et al. [40] evaluated the immunohistochemical expressions of p16 and cyclin D1 in 224 cases and observed that p16/cyclin D1 indexes were significantly higher in HER2-positive and TNBC groups than in a luminal group and that this index was associated with poor prognosis. Pare et al. [28] investigated DCR2 expression in 1,032 tissues by IHC and reported DCR2 overexpression was associated with poor prognostic factors such as high histologic grade, ER negativity, PR negativity, and HER2 positivity. In addition, they found DCR2 overexpression was not related to age, tumor size, stage, or LVI, and reported that the DCR2 positive group tended to have poorer prognoses than the DCR2 negative group.

In the present study, SASP positivity was associated with poorer prognosis in the luminal A group and multivariate analysis showed SASP positivity independently predicted BCSS and DFS, and these results were broadly in-line with several previous studies. Kim et al. [41] investigated the role of cellular senescence in papillary thyroid carcinoma (PTC) and found that senescent tumor cells were present at the invasive borders of PTC and exhibited a higher invasive ability than non-senescent tumor cells by upregulated MMPs. They also demonstrated that senescent cancer cells lead the collective invasion through CXCL12/CXCR4 signaling and enhance collective LVI in thyroid cancer. Similar result was obtained in our study. LVI was more frequently observed in SASP-positive group than in SASP-negative group in luminal A .

Inflammatory cytokines secreted by senescent tumor cells can modulate tumor microenvironment. IL- 6 was known to activate STAT3 and consequently increase MMP-2 expression and the metastatic ability of cancer cells in malignant melanoma [42]. Waugh and Wilson [43] reported IL-8 secreted by tumor cells plays an important role in the tumor microenvironment and observed that the expressions of the IL- 8 receptors, CXCR1 and CXCR2, were elevated in cancer cells, endothelial cells, and tumorinfiltrating neutrophils and macrophages. The authors concluded IL-8 signaling increases cancer cell proliferation and survival, promotes angiogenesis by stimulating endothelial cells, and promotes the migration of neutrophils into tumor tissues and their activations. Vascular endothelial growth factor (VEGF, a SASP factor) is secreted by senescent cells and promotes cancer cell growth by promoting angiogenesis and facilitating access to growth factors [44]. Although we did not investigate the associations between SASP and tumor tissue concentrations of MMP, IL-6, or IL-8, we believe that the significantly higher frequency of LVI observed in SASP-positive tumors reflected increased tumor cell invasiveness induced by factors secreted by senescent tumor cells.

In tumor microenvironments, IL-6 signaling not only promotes proliferation and survival but also has anti-tumor effects due to inhibition of tumor growth through T-cell immune response. IL-6 promotes $\mathrm{T}$ cell migration to lymph nodes and tumor areas and can exhibit cytotoxic effects on tumor cells by activating $\mathrm{T}$ cells [45]. In the present study, SASP was significantly associated with high sTIL density and high iCD103 + lymphocyte levels. Although we did not measure IL-6 secreted by SASP-positive cells, we think that high sTIL density and high iCD103 + lymphocyte levels in SASP-positive tumors would be, in part, $\mathrm{T}$ cell migration to tumor site by IL- 6 signaling by senescent cells. In contrast to that observed in the luminal A subtype, SASP positivity was associated with good prognosis in TNBC. In our previous studies, high sTIL density and high iCD103 + lymphocyte counts were good prognostic factors in a subset of s (e.g., TNBC) and iCD103 + lymphocyte infiltration was more closely related to prognosis than sTIL density [30]. In the present study, SASP was not related to sTIL density and iCD103 + lymphocyte count was significantly higher in SASP-positive cases in TNBC. These relationships may, in part, explain the better prognosis of SASP-positive tumors in TNBC. However, we suggest further study be conducted on relationships between biomarker expressions (ER, PR, and HER2) and biologic effects of SASP.

This study has several limitations. It is a single-center and retrospective study and immunohistochemical expression of SASP was observed in TMA tissues rather than in whole 
sections. Furthermore, we did not investigate SASP factors by placing focus on their functions. However, we conducted this study on a large cohort of primary IBCs with follow-up data.

Summarizing, immunohistochemically detected SASP in tissues was significantly associated with tumor aggressiveness (LVI, high histologic grade, and triple-negativity) and with immune microenvironments exhibiting high sTIL density and iCD103 + lymphocyte infiltration. In addition, the prognostic significance of SASP was found to be dependent on the surrogate molecular subtypes of breast cancer.

\section{DATA AVAILABILITY STATEMENT}

The data that support the findings of this study are available from the corresponding author upon reasonable request. Requests to access the datasets should be directed to Young Kyung Bae, ykbae@ynu.ac.kr.

\section{ETHICS STATEMENT}

The studies involving human participants were reviewed and approved by the Institutional Review Board (IRB) of Yeungnam University Medical Center (YUMC2019-10-002). Written informed consent for participation was not required for this

\section{REFERENCES}

1. Childs BG, Gluscevic M, Baker DJ, Laberge R-M, Marquess D, Dananberg J, et al. Senescent Cells: an Emerging Target for Diseases of Ageing. Nat Rev Drug Discov (2017) 16(10):718-35. doi:10.1038/nrd.2017.116

2. Basisty N, Kale A, Jeon OH, Kuehnemann C, Payne T, and Rao CA. Proteomic Atlas of Senescence-Associated Secretomes for Aging Biomarker Development. Plos Biol (2020) 18(1):e3000599. doi:10.1371/journal.pbio.3000599

3. Coppé J-P, Patil CK, Rodier F, Sun Y, Muñoz DP, Goldstein J, et al. Senescence-associated Secretory Phenotypes Reveal Cell-Nonautonomous Functions of Oncogenic RAS and the P53 Tumor Suppressor. Plos Biol (2008) 6(12):e301-2868. doi:10.1371/journal.pbio.0060301

4. Coppé J-P, Desprez P-Y, Krtolica A, and Campisi J. The Senescence-Associated Secretory Phenotype: the Dark Side of Tumor Suppression. Annu Rev Pathol Mech Dis (2010) 5:99-118. doi:10.1146/annurev-pathol-121808-102144

5. Kang T-W, Yevsa T, Woller N, Hoenicke L, Wuestefeld T, Dauch D, et al. Senescence Surveillance of Pre-malignant Hepatocytes Limits Liver Cancer Development. Nature (2011) 479(7374):547-51. doi:10.1038/nature10599

6. Krizhanovsky V, Xue W, Zender L, Yon M, Hernando E, and Lowe SW. Implications of Cellular Senescence in Tissue Damage Response, Tumor Suppression, and Stem Cell Biology. Cold Spring Harbor Symposia Quantitative Biol (2008) 73:513-22. doi:10.1101/sqb.2008.73.048

7. Di Mitri D, and Alimonti A. Non-Cell-Autonomous Regulation of Cellular Senescence in Cancer. Trends Cel Biol (2016) 26(3):215-26. doi:10.1016/ j.tcb.2015.10.005

8. Biran A, Zada L, Abou Karam P, Vadai E, Roitman L, Ovadya Y, et al. Quantitative Identification of Senescent Cells in Aging and Disease. Aging Cell (2017) 16(4):661-71. doi:10.1111/acel.12592

9. Collado M, and Serrano M. The Power and the Promise of Oncogene-Induced Senescence Markers. Nat Rev Cancer (2006) 6(6):472-6. doi:10.1038/nrc1884

10. Davalos AR, Kawahara M, Malhotra GK, Schaum N, Huang J, Ved U, et al. p53-dependent Release of Alarmin HMGB1 Is a central Mediator of Senescent Phenotypes. J Cel Biol (2013) 201(4):613-29. doi:10.1083/jcb.201206006 study in accordance with the national legislation and the institutional requirements.

\section{AUTHOR CONTRIBUTIONS}

Conceptualization: YB, JK. Funding acquisition: JK. Investigation: MP, JC, and YB. Supervision: YB, JK. Writing-original draft: MP, YB. Writing-review and editing: YB, JC, and JK. Approval of the final manuscript: all authors.

\section{FUNDING}

This study was supported by Medical Research Center Program (2015R1A5A2009124) through the National Research Foundation of Korea (NRF), Ministry of Science, ICT, and Future Planning.

\section{CONFLICT OF INTEREST}

The authors declare that the research was conducted in the absence of any commercial or financial relationships that could be construed as a potential conflict of interest.

11. Wiley CD, Velarde MC, Lecot P, Liu S, Sarnoski EA, Freund A, et al. Mitochondrial Dysfunction Induces Senescence with a Distinct Secretory Phenotype. Cel Metab (2016) 23(2):303-14. doi:10.1016/j.cmet.2015.11.011

12. Zhang Z, Rosen DG, Yao JL, Huang J, and Liu J. Expression of p14ARF, p15INK4b, p16INK4a, and DCR2 Increases during Prostate Cancer Progression. Mod Pathol (2006) 19(10):1339-43. doi:10.1038/modpathol.3800655

13. Nobori T, Miura K, Wu DJ, Lois A, Takabayashi K, and Carson DA. Deletions of the Cyclin-dependent Kinase-4 Inhibitor Gene in Multiple Human Cancers. Nature (1994) 368(6473):753-6. doi:10.1038/368753a0

14. Stone S, Jiang P, Dayananth P, Tavtigian SV, Katcher H, Parry D, et al. Complex Structure and Regulation of the P16 (MTS1) Locus. Cancer Res (1995) 55(14):2988-94.

15. Krishnamurthy J, Ramsey MR, Ligon KL, Torrice C, Koh A, Bonner-Weir S, et al. p16INK4a Induces an Age-dependent Decline in Islet Regenerative Potential. Nature (2006) 443(7110):453-7. doi:10.1038/nature05092

16. Günakan E, Tohma YA, Karakaș LA, Akıllı H, Haberal AN, and Ayhan A. Prognostic Impact of P16 and P53 Gene Expressions in Stage 1a Epithelial Ovarian Cancer. Obstet Gynecol Sci (2020) 63(4):464-9. doi:10.5468/ogs.19204

17. Kida K, Terada T, Uwa N, Omori Y, Fujii T, Tomita Y, et al. Relationship between P16 Expression and Prognosis in Patients with Oropharyngeal Cancer Undergoing Surgery. In Vivo (2018) 32(4):927-35. doi:10.21873/invivo.11331

18. Reimers N, Kasper HU, Weissenborn SJ, Stützer H, Preuss SF, Hoffmann TK et al. Combined Analysis of HPV-DNA, P16 and EGFR Expression to Predict Prognosis in Oropharyngeal Cancer. Int J Cancer (2007) 120(8):1731-8. doi:10.1002/ijc.22355

19. Malumbres M, Pérez De Castro I, Hernández MI, Jiménez M, Corral T, and Pellicer A. Cellular Response to Oncogenic Ras Involves Induction of the Cdk4 and Cdk6 Inhibitor P15 INK4b. Mol Cel Biol (2000) 20(8):2915-25. doi:10.1128/mcb.20.8.2915-2925.2000

20. Liu X, Yue P, Khuri FR, and Sun S-Y. Decoy Receptor 2 (DcR2) Is a P53 Target Gene and Regulates Chemosensitivity. Cancer Res (2005) 65(20):9169-75. doi:10.1158/0008-5472.Can-05-0939

21. Bianchi ME. DAMPs, PAMPs and Alarmins: All We Need to Know about Danger. J Leukoc Biol (2007) 81(1):1-5. doi:10.1189/jlb.0306164 
22. Völp K, Brezniceanu ML, Bösser S, Brabletz T, Kirchner T, Göttel D, et al. Increased Expression of High Mobility Group Box 1 (HMGB1) Is Associated with an Elevated Level of the Antiapoptotic C-IAP2 Protein in Human colon Carcinomas. Gut (2006) 55(2):234-42. doi:10.1136/gut.2004.062729

23. Yamada S, and Maruyama I. HMGB1, a Novel Inflammatory Cytokine. Clinica Chim Acta (2007) 375(1-2):36-42. doi:10.1016/j.cca.2006.07.019

24. Jeon OH, Kim C, Laberge R-M, Demaria M, Rathod S, Vasserot AP, et al. Local Clearance of Senescent Cells Attenuates the Development of post-traumatic Osteoarthritis and Creates a Pro-regenerative Environment. Nat Med (2017) 23(6):775-81. doi:10.1038/nm.4324

25. Victorelli S, Lagnado A, Halim J, Moore W, Talbot D, Barrett K, et al. Senescent Human Melanocytes Drive Skin Ageing via Paracrine Telomere Dysfunction. Embo j (2019) 38(23):e101982. doi:10.15252/embj.2019101982

26. Brezniceanu M-L, Völp K, Bösser S, Solbach C, Lichter P, Joos S, et al. HMGB1 Inhibits Cell Death in Yeast and Mammalian Cells and Is Abundantly Expressed in Human Breast Carcinoma. FASEB j. (2003) 17(10):1295-7. doi:10.1096/fj.02-0621fje

27. Choi YR, Kim H, Kang HJ, Kim NG, Kim JJ, Park KS, et al. Overexpression of High Mobility Group Box 1 in Gastrointestinal Stromal Tumors with KIT Mutation. Cancer Res (2003) 63(9):2188-93.

28. Pare R, Soon PS, Shah A, and Lee CS. Differential Expression of Senescence Tumour Markers and its Implications on Survival Outcomes of Breast Cancer Patients. PLoS One (2019) 14(4):e0214604. doi:10.1371/journal.pone.0214604

29. Kim MC, Park MH, Kang SH, and Bae YK. NDRG3 Protein Expression Is Associated with Aggressive Biologic Phenotype and Unfavorable Outcome in Patients with Invasive Breast Cancer. Int J Clin Exp Pathol (2019) 12(10):3886-93.

30. Park MH, Kwon SY, Choi JE, Gong G, and Bae YK. Intratumoral CD103positive Tumour-infiltrating Lymphocytes Are Associated with Favourable Prognosis in Patients with Triple-negative Breast Cancer. Histopathology (2020) 77:560-9. doi:10.1111/his.14126

31. Kwon HJ, Choi JE, Kang SH, Son Y, and Bae YK. Prognostic Significance of CD9 Expression Differs between Tumour Cells and Stromal Immune Cells, and Depends on the Molecular Subtype of the Invasive Breast Carcinoma. Histopathology (2017) 70(7):1155-65. doi:10.1111/his.13184

32. Hammond MEH, Hayes DF, Dowsett M, Allred DC, Hagerty KL, Badve S, et al. American Society of Clinical Oncology/College of American Pathologists Guideline Recommendations for Immunohistochemical Testing of Estrogen and Progesterone Receptors in Breast Cancer. Jco (2010) 28(16):2784-95. doi:10.1200/jco.2009.25.6529

33. Wolff AC, Hammond MEH, Allison KH, Harvey BE, Mangu PB, Bartlett JMS, et al. Human Epidermal Growth Factor Receptor 2 Testing in Breast Cancer: American Society of Clinical Oncology/College of American Pathologists Clinical Practice Guideline Focused Update. Jco (2018) 36(20):2105-22. doi:10.1200/jco.2018.77.8738

34. Goldhirsch A, Winer EP, Coates AS, Gelber RD, Piccart-Gebhart M, Thürlimann B, et al. Personalizing the Treatment of Women with Early
Breast Cancer: Highlights of the St Gallen International Expert Consensus on the Primary Therapy of Early Breast Cancer 2013. Ann Oncol (2013) 24(9): 2206-23. doi:10.1093/annonc/mdt303

35. Jang N, Kwon HJ, Park MH, Kang SH, and Bae YK. Prognostic Value of Tumor-Infiltrating Lymphocyte Density Assessed Using a Standardized Method Based on Molecular Subtypes and Adjuvant Chemotherapy in Invasive Breast Cancer. Ann Surg Oncol (2018) 25(4):937-46. doi:10.1245/ s10434-017-6332-2

36. Salgado R, Denkert C, Demaria S, Sirtaine N, Klauschen F, Pruneri G, et al. The Evaluation of Tumor-Infiltrating Lymphocytes (TILs) in Breast Cancer: Recommendations by an International TILs Working Group 2014. Ann Oncol (2015) 26(2):259-71. doi:10.1093/annonc/mdu450

37. Wang Z-Q, Milne K, Derocher H, Webb JR, Nelson BH, and Watson PH. CD103 and Intratumoral Immune Response in Breast Cancer. Clin Cancer Res (2016) 22(24):6290-7. doi:10.1158/1078-0432.Ccr-16-0732

38. Webb JR, Milne K, Watson P, Deleeuw RJ, and Nelson BH. Tumorinfiltrating Lymphocytes Expressing the Tissue Resident Memory Marker CD103 Are Associated with Increased Survival in High-Grade Serous Ovarian Cancer. Clin Cancer Res (2014) 20(2):434-44. doi:10.1158/10780432.Ccr-13-1877

39. Liu Z, Wang L, Yang J, Bandyopadhyay A, Kaklamani V, Wang S, et al. Estrogen Receptor Alpha Inhibits Senescence-like Phenotype and Facilitates Transformation Induced by Oncogenic Ras in Human Mammary Epithelial Cells. Oncotarget (2016) 7(26):39097-107. doi:10.18632/oncotarget.9772

40. Kim GJ, Kim DH, Min KW, and Kim SH. Prognostic Impact of High P16/ cyclin D1 index in Breast Cancer. Int J Clin Exp Pathol (2019) 12(6):2224-32.

41. Kim YH, Choi YW, Lee J, Soh EY, Kim J-H, and Park TJ. Senescent Tumor Cells lead the Collective Invasion in Thyroid Cancer. Nat Commun (2017) 8: 15208. doi: $10.1038 /$ ncomms 15208

42. Xie T-x., Wei D, Liu M, Gao AC, Ali-Osman F, Sawaya R, et al. Stat3 Activation Regulates the Expression of Matrix Metalloproteinase-2 and Tumor Invasion and Metastasis. Oncogene (2004) 23(20):3550-60. doi:10.1038/sj.onc.1207383

43. Waugh DJJ, and Wilson C. The Interleukin-8 Pathway in Cancer. Clin Cancer Res (2008) 14(21):6735-41. doi:10.1158/1078-0432.Ccr-07-4843

44. Carmeliet P. VEGF as a Key Mediator of Angiogenesis in Cancer. Oncology (2005) 69(Suppl. 3):4-10. doi:10.1159/000088478

45. Fisher DT, Appenheimer MM, and Evans SS. The Two Faces of IL-6 in the Tumor Microenvironment. Semin Immunol (2014) 26(1):38-47. doi:10.1016/ j.smim.2014.01.008

Copyright (C) 2021 Park, Choi, Kim and Bae. This is an open-access article distributed under the terms of the Creative Commons Attribution License (CC BY). The use, distribution or reproduction in other forums is permitted, provided the original author(s) and the copyright owner(s) are credited and that the original publication in this journal is cited, in accordance with accepted academic practice. No use, distribution or reproduction is permitted which does not comply with these terms. 